http://dx.doi.org/10.21611/qirt.1994.001

\title{
Spatial resolution enhancement of an IR system by image restoration techniques
}

\author{
by BOUGEARD D., VERMEULEN J.P. and BAUDOIN B.
}

\begin{abstract}
Département Energétique Industrielle, Ecole des mines de Douai, 941, Rue Charles Bourseul, BP 838, Douai Cedex, France.
\end{abstract}

\section{Abstract}

The spatial resolution of infrared systems is one of the major limitations in the use of IR thermography for temperature measurements, mainly in the case of small objects of size [1]. The use of digital image restoration techniques for spatial resolution enhancement is a very powerful method to reduce this limitation. Two restoration filters are examined in this paper. The accuracy of these two mothods is based on the accurate determination of the Optical Transfer Function (OTF) and of the noise spectral density.

\section{Nomenclature}

$\begin{array}{ll}f(x, y) & \text { Object function ( } x, y \text { co-ordinates in the object plane) } \\ F(u, v) & \text { Fourier transformation of } f \\ g(x, y) & \text { Image function (x,y co-ordinates in the image plane) } \\ G(u, v) & \text { Fourier transformation of } g \\ h(x, y) & \text { Point Spread Function } \\ H(u, v) & \text { Optical Transfer Function } \\ M(u, v) & \text { Wiener filter } \\ S_{\mathrm{M}}(u, v) & \text { Spectral density of the non degraded signal } \\ S_{v v}(u, v) & \text { Spectral density of noise }\end{array}$

\section{Introduction}

Image restoration deals with what has been recorded in the presence of one or more sources of degradation. There are many sources of degradation in IR imaging systems :

- some types of degradation affect only the thermal level of the individual picture points without introducing spatial blur. This degradation is generated by the detector noise itself and by the 12 bit digitization of the signal by our acquisition system.

- other types which do involve blur are called spatial degradation. These types are a consequence of the spatial averaging effect over the IPA (Instantaneous Projected Area), and also by optical diffraction and aberration blur (for example defocusing)[2].

Given an ideal picture $f(x, y)$ and the corresponding degraded picture $g(x, y)$, we can assume that $g$ and $f$ are related by:

$$
g(x, y)=\iint h\left(x, y, x^{\prime}, y^{\prime}\right) f\left(x^{\prime}, y^{\prime}\right) d x^{\prime} d y^{\prime}+v(x, y)
$$

where $h\left(x, y, x^{\prime}, y^{\prime}\right)$, called the point spread function (PSF), is the degradation function that determines the radiant energy distribution in the image plane $(x, y)$ due to a point source of radiant energy located in the object plane $\left(x^{\prime}, y^{\prime}\right) ;(x, y)$ is the random noise (assumed to be additive) that may be present in the output picture.

If we make the assumption that the PSF is independent of the position, equation (1) becomes: 


$$
g(x, y)=\iint h\left(x-x^{\prime} y-y^{\prime}\right) f\left(x^{\prime}, y^{\prime}\right) d x^{\prime} d y^{\prime}+u(
$$

\section{Inverse filtering}

If we assume that the noise is negligible, using the convolution theorem the Fourier transformation of equation (2) gives, :

$$
\mathrm{G}(\mathrm{u},
$$

Where $G(u, v), H(u, v), F(u, v)$

The function $\mathrm{H}($

$f(x, y)$ into the degraded picture $g(x, y)$. So equation (3) implies that if $H($ restore $f(x, y)$ by multiplying the Fourier transform $G(u, v)$ of the degraded picture by $1 / H($ and then perform an inverse Fourier transformation. A number of practical prublems arise when one attempts to make use of (3); in particular a larger amplification of high spatial frequency noise occurs [4]. The solution is to restore only the frequencies in the $u, v$ plane where the signal to noise ratio is high. For that we can limit the inverse filter to low spatial frequencies [3] for a single field, or we can average over several fields in order to decrease noise. This last solution obviously implies that the thermal scene is independent of time. In each case, the problem is to determine the OTF.

\section{OTF measurement}

In some cases (diffraction in optics) it is possible to determine the OTF from the theory of degradation. Here the degradation phenomenon is very complex, so we try to measure directly the OTF. Two methods are experimented. First we measure the vertical and horizontal line spread functions [5]. The test target is a narrow slit placed in front of a uniform thermal black body. The noise is limited by an average taken over 200 fields. Applying a Fourier transformation to the image and the object we can determine from equation (3) $\mathrm{H}$ ( $\mathrm{H}(0, \mathrm{v})$ which are respectively the horizontal and the vertical line transfer functions (the Fourier transformation of the object being mathematically known). In a first approximation we can take the product of these two functions to create the two-dimensional transfer function.

The second method is to determine the PSF by viewing a very narrow circular hole in front of a black body and to take the Fourier transformation of the image. If the hole is small enough, the result is close to the real PSF. Here, the experimental difficulty is to avoid the output signal getting lost in the noise, so this time we average over 300 fields. The twodimensional OTF measured by the two methods is very close (less than $5 \%$ difference).

Figures $1 \mathrm{a}$ and $1 \mathrm{~b}$ represent the vertical and horizontal line transfer functions

\section{Wiener filter}

To restore images in the presence of noise, we use a Wiener filter [8]. This is a well documented filter determined by:

$$
M(u, v)=\frac{1}{H(u, v)} \times \frac{|H(u, v)|^{2}}{|H(u, v)|^{2}+\left[\frac{S_{v v}(u, v)}{S_{\text {fr }}(u, v)}\right]}
$$


http://dx.doi.org/10.21611/qirt.1994.001

where $S_{v v}(u, v)$ is the noise power density spectrum. This quantity is measured using the difference between a single field and the resulting time-averaged field.

\section{Experimental results}

\subsection{Inverse filtering}

To measure the performance of this method we measure the spot response function of the camera. A variable circular hole is placed in front of a black body and we measure the modulation of the output signal as a function of the hole width [1] [7] (cf. figure 2). Figure 3 shows this function with and without the inverse filter. We can see from this curve that the solid angle ,giving $95 \%$ modulation, is divided by 4 in the case of the restored image, this means that the spatial resolution is enhanced by a factor 4 . On figure 4, we show this enhancement of spatial resolution, by targeted heated letters with and without using our filter. The images clearly show the benefit of the method.

\subsection{Wiener filter}

Figure $5 a$ presents a single line profile, which has to be compared with figures $5 b$ and $5 c$ which present the restored profiles with respectively the inverse filter and the Wiel,er filter. The comparison between figure $5 b$ and $5 c$ clearly show the advantage of the Wiener filter when the noise is large compared to the signal.

\section{Conclusions}

In this paper, we show that the restoration of a degraded infrared image is possible even in the presence of noise. The results also show that we can enhance by $400 \%$ (at $95 \%$ modulation) the spatial resolution of infrared cameras with these methods.

\section{REFERENCES}

[1] PAJANI (D.) - Mesure par thermographie infrarouge. 1989 ADD, Editeur.

[2] AZRIEL ROSENFELD and AVINASH (C.) KAK. - Digital image processing - second edition. 1982 , by Academic press, Inc.

[3] ANDREWS (H.C.), HUNT (B.R.). - Digital image restoration. 1977 Prentice Hall Inc.

[4] THOMAS (G.). - Reconstitution de signaux déformés par un système linéaire. Thèse de I'Université Claude Bernard de Lyon 1981.

[5] ARGIRIOU. (A.) - La mesure en thermographie infrarouge, calibration et traitement du șignal: application à la validation d'une modélisation numérique, en régime instationnaire, d'un convertisseur de type THEK. Thèse de l'Universitè de Provence 1987.

[6] BOUGEARD (D.) - Augmentation du transfert thermique à l'aide de promoteurs de lurbulence: Mise au point du dispositif de thermographie infrarouge d'un banc experimental. DEA de l'Université de Valenciennes et du hainaut Cambrésis 1993.

[7] WILLIAMS (D.) - The MTF of thermal imaging cameras - its relevance and measurements (QIRT 92).

[8] PEARCE (J.) CHULZE and RYU (Z.) Improving the accuracy of inferred temperatures in small spot size experiments (QIRT92). 
http://dx.doi.org/10.21611/qirt.1994.001
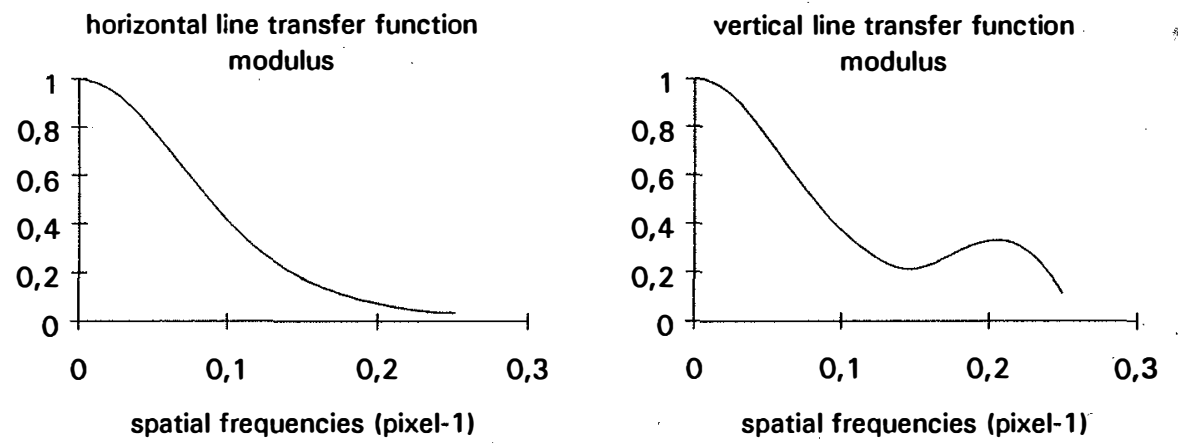

Fig. 1 - a) Horizontal and b) vertical line transfer functions modulus.

uniform black body (thermal level $\mathrm{n} 1$ )

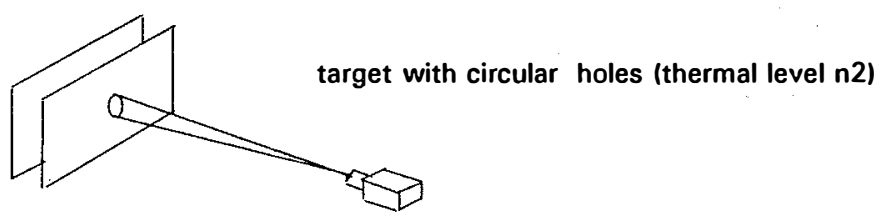

camera response (thermal level $n^{\prime} 1$ )

modulation $=\left(n^{\prime} 1-n 2\right) /((n 1-n 2)$

Fig. 2 - Spot Response Function measurements.

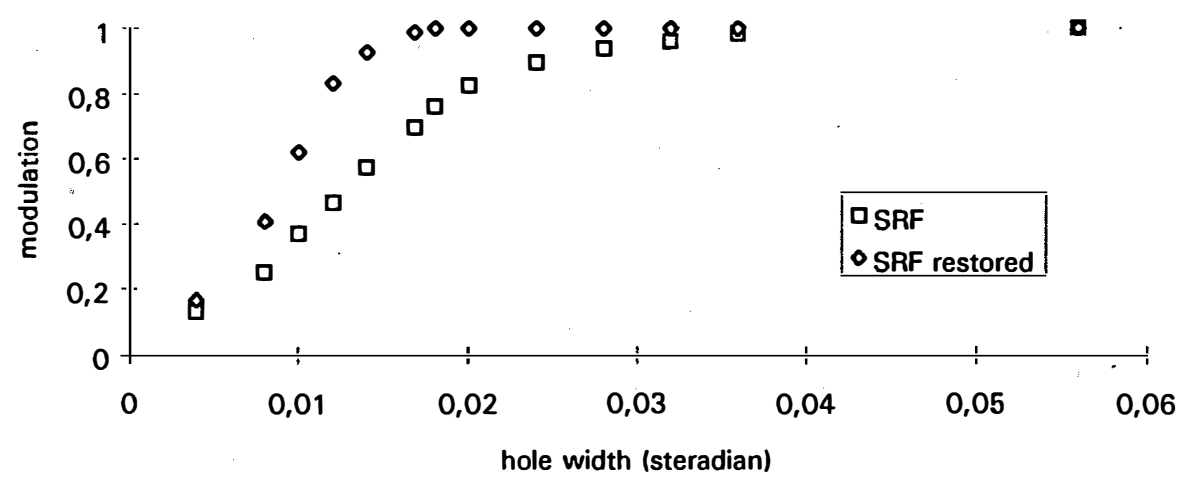

Fig. 3 - Spot Response Function with and without the inverse filter. 
http://dx.doi.org/10.21611/qirt.1994.001

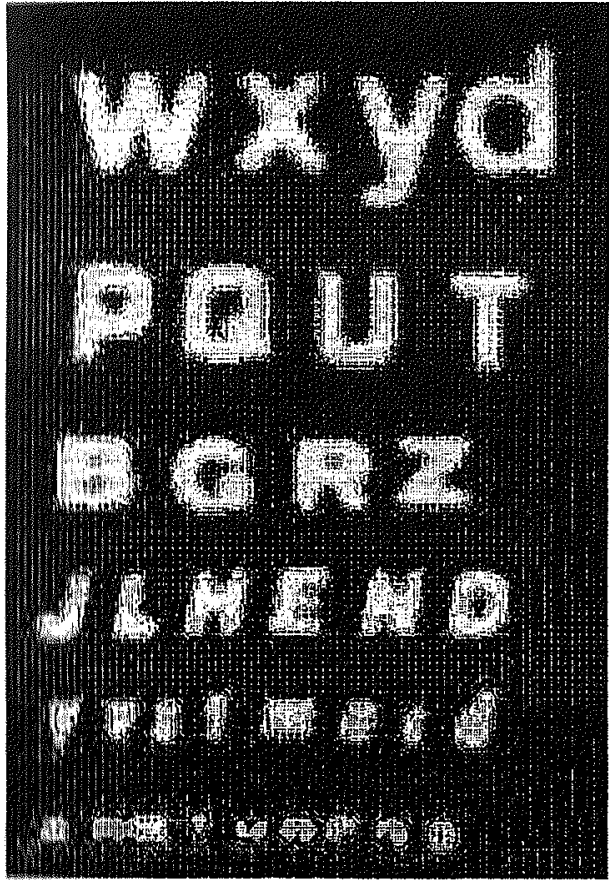

(a)

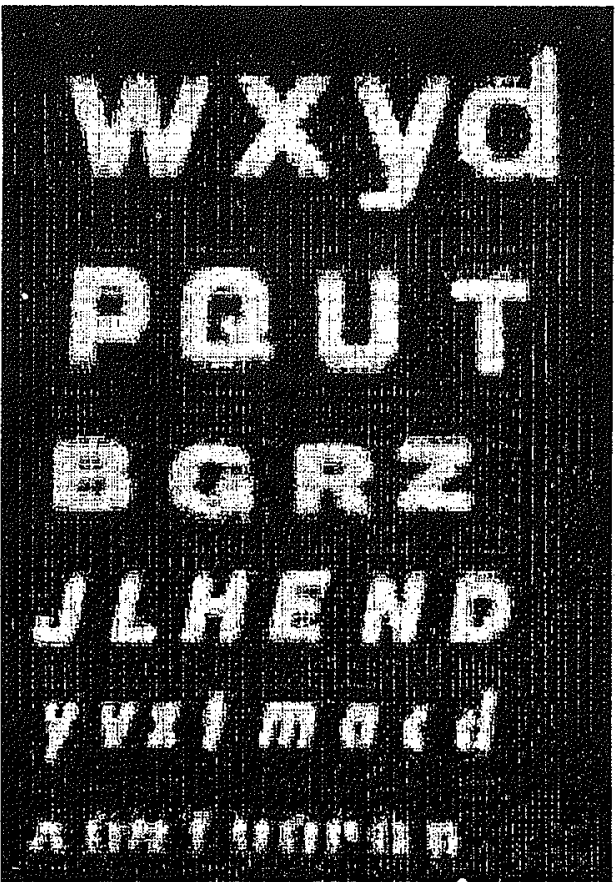

(b)

Fig. 4 - Heated letters viewed with IR camera (a) and restored with the inverse filter (b). 
http://dx.doi.org/10.21611/qirt.1994.001
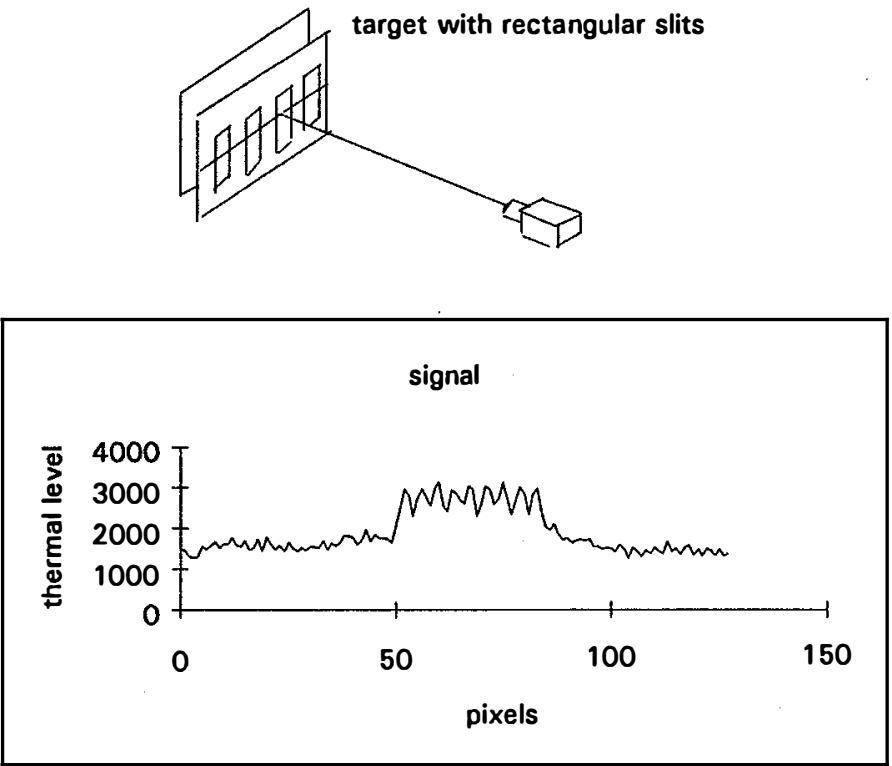

(a)

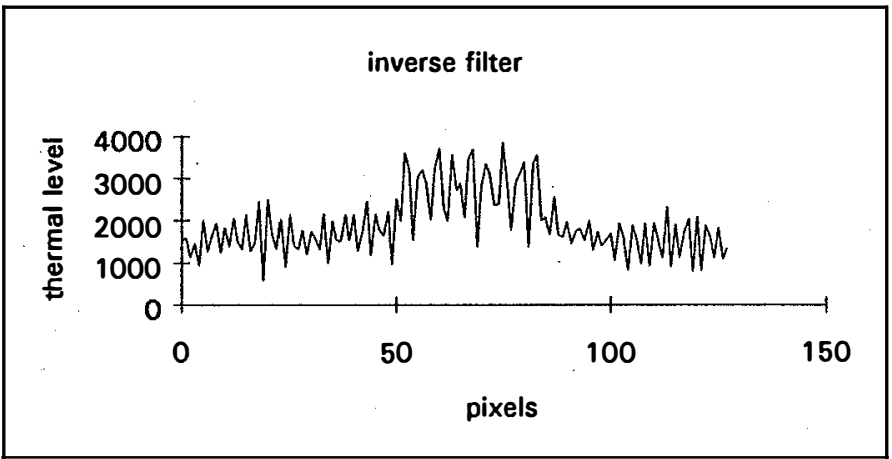

(b)

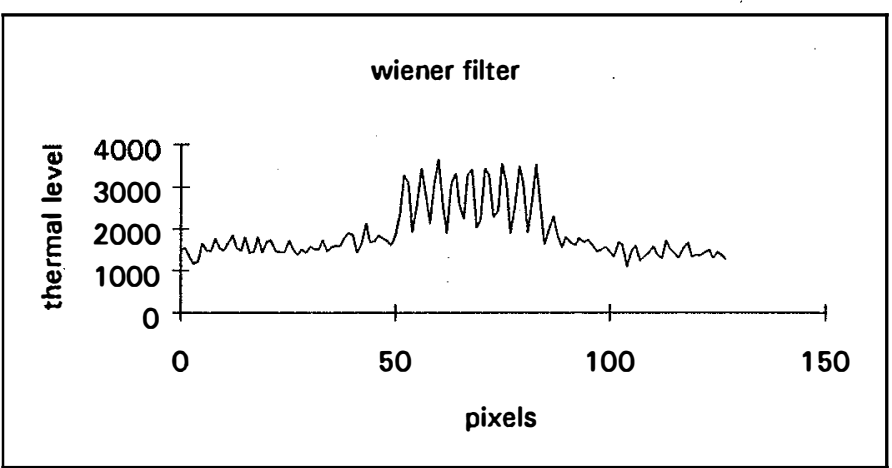

(c)

Fig. 5 - Comparison of a single line profile restored with the inverse filter and the wiener filter 